CERN-PH-TH/2005-199

DISTA-UPO-05

\title{
N=2 Superparticles, RR Fields, and Noncommutative Structures of (super)-Spacetime ${ }^{1}$
}

\author{
Pietro Antonio Grassi \\ Centro Studi e Ricerche E. Fermi, Compendio Viminale, I-00184, Roma, Italy, \\ CERN, Theory Unit, Physics Department, 1211-CH Geneva 23, Switzerland, \\ DISTA, Università del Piemonte Orientale, via Bellini 25/g, I-15100, Alessandria, Italy. \\ pietro.grassi@cern.ch
}

December 22, 2013

\begin{abstract}
The recent developments in superstring theory prompted the study of non-commutative structures in superspace. Considering bosonic and fermionic strings in a constant antisymmetric tensor background yields a non-vanishing commutator between the bosonic coordinates of the spacetime. Likewise, the presence of constant Ramond-Ramond (RR) background leads to a non-vanishing anti-commutator for the Grassmann coordinates of the superspace. The non-vanishing commutation relation between bosonic coordinates can also be derived using a particle moving in a magnetic background, we use $\mathrm{N}=2$ pure spinor superparticles and $D 0$-branes to show how the non-commutative structures emerge in superspace. It is argued how a $D 0$-brane in a background of $\mathrm{RR}$ fields reproduces the results obtained in string theory.
\end{abstract}

\footnotetext{
${ }^{1}$ Based on a lecture given at $43^{\text {rd }}$ International School of Subnuclear Physics, Erice, Sicily, Italy, Aug. 2005.
} 


\section{Contents}

\begin{tabular}{lll}
\hline Introduction & 2
\end{tabular}

2 A Particle in a Magnetic Field 5

3 Superparticles and the BRST svmmetrv 5

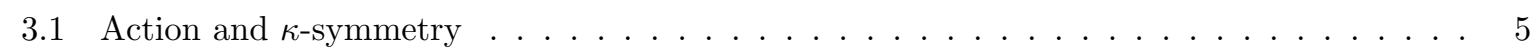

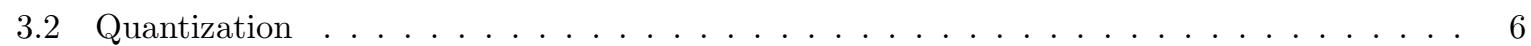

3.3 Coupling the superparticle to the background $\ldots \ldots \ldots \ldots \ldots \ldots \ldots$

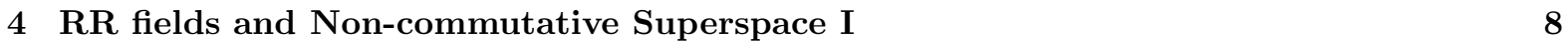

4.1 Radiative Corrections . . . . . . . . . . . . . . . . . . . . . . . . . . . 11

$\begin{array}{lll}5 & \text { D0-branes and the BRST svmmetry } & 11\end{array}$

$\begin{array}{lll}6 & \text { RR fields and Non-commutative Superspace II } & 12\end{array}$

\section{Introduction}

During the last years, several new ideas emerged from the marriage of non-commutative geometry to quantum field theory and string theory. This is due to the discovery that the spacetime generated by strings propagating on a non-trivial background [1, 2, 3, 4, 5, is non-commutative. Furthermore string theory provides a meaningful way to construct quantum field theories on non-commutative spaces.

Nevertheless the history of non-commutative geometry and non-commutative structures of spacetime is definitely longer and it has its roots in quantum mechanics. Indeed, it was first realized by Peierls using non-relativistic quantum mechanics that the motion of a charged particle in presence of non-trivial external magnetic fields can be described by a free Hamiltonian assuming non-vanishing commutation relations for the coordinates [6, 7, 8].

The simplest example of non-commutative spacetime [9, 10] is represented by the Heisenberg algebra of the coordinates

$$
\left[x^{m}, x^{n}\right]=i \theta^{m n}
$$

where $\theta^{m n}$ is a constant antisymmetric tensor. Based on this prototype, it has been developed an enormous amount of new mathematics which we are not going to review here. The commutation relations (1.1) can be recovered from the Hamiltonian of a charged particle moving in a background of magnetic field in the massless limit $m \rightarrow 0$. In that limit the particle is confined in the lowest Landau level and this limit can consistently be taken if some constraints on the momenta are imposed. These are second-class constraints that have to be treated using the Dirac brackets and this yields the commutation relations (1.1). We briefly review this model in Sec. 2. Then, we move to the supersymmetric version.

As is well-known, bosonic particles and bosonic string theories are not sufficient to provide a complete description of particle physics since they do not include fermionic degrees of freedom (except maybe only for some unphysical ghost fields). Thus, we have to extend the bosonic theory to a fermionic one. There are essentially two ways to do it: adding some fermionic (anticommuting) worldline spinors $\psi^{m}$ (or worldsheet spinor in the case of superstrings) or 
adding some fermionic target-space spinors $\theta^{\alpha}$ [11, 12]. In the former case supersymmetry on the worldsheet has to be imposed for a consistent formulation of the model, whereas for the latter case, one has to impose a new gauge symmetry, known as $\kappa$-symmetry [13], and this leads to supersymmetry in the target space. We recall the basic ingredients of this superparticle model in Sec. 3 .

The quantization of superparticle is unfortunately very problematic. The action is obtained from the bosonic one by replacing the momentum $\Pi^{m}$ with its supersymmetric version $\Pi^{m}=$ $\dot{x}^{m}+\theta \gamma^{m} \dot{\theta}$ and this leads to fermionic constraints since the momentum $p_{\alpha}$ is algebraically related to its conjugated variable $\theta^{\alpha}$. However, these constraints mix first-class constraints which generate the $\kappa$-symmetry - with second-class constraints and there is no Lorentz-covariant way to separate the twos. Several procedures were conceived to covariantly quantize these models (see for example [14 and the references therein), but most of them were nonpractical for computations and were abandoned. ${ }^{2}$

On the other hand, the recent work by N. Berkovits [16] provides a new technique to handle the quantization of the superparticle and the superstring theory. In this new framework, the action of the superparticle is replaced by a free action and the physical states are constructed using a BRST charge acting on the Hilbert space of free fields. To be more precise, some of fields are not really free. Indeed, to define a BRST charge $Q$, one has to introduce new degrees of freedom which play the role of ghosts here denoted by $l^{\alpha}$, and the nilpotency of $Q$ implies the quadratic constraints

$$
l^{\alpha} \gamma_{\alpha \beta}^{m} l^{\beta}=0,
$$

where $\gamma_{\alpha \beta}^{m}$ are the Dirac matrices in the Majorana basis and they are symmetric (in 10 dimensions). The spinors satisfying eq. (1.2) are known as pure spinors and the formalism is now denoted as Pure-Spinor Formulation. Here, we consider only the Pure Spinor formulation of superparticle and D0-branes and we refer to [17, 18, 19. It has been also considered the possibility to remove the constraint by adding new ghost fields in the work [22, 23, 24] and in the specific case of superparticle this was explored in [25]. However for the purposes of the present work we will use the Pure-Spinor formulation whose basic ingredients will be reviewed in Sec. 3.2. Thus, given a consistent way to quantize the superparticle we can study the spectrum and the interactions.

At the massless (lowest) level string theory can be described by an effective theory of supergravity and the spectrum consists of a bosonic sector with the graviton $G_{m n}$, the NS-NS antisymmetric tensor $B_{m n}$, the dilaton $\phi$ and a set of $p$-forms $F_{p}{ }^{3}$, and a fermionic sector, a.k.a. NS-R or R-NS sector, which contains the gravitinos $\Psi_{m}^{\alpha}$ (see [26] for a complete reference). The interest of superparticles in this context is due to the fact that they can be viewed as truncations of string theory to the massless sector. Therefore these models are useful to deduce some general aspects of string theory such as the spectrum of the massless modes, their equations of motion, and some radiative corrections, even if they can be used only a limited amount of amplitude computations [27, 28.

To be more precise, the $\mathrm{N}=1 \mathrm{~d}=10$ superparticle describe the multiplet of $\mathrm{N}=1$ super-YangMills theory. The spectrum is characterized by the gluon ( 8 on-shell dofs) and the gluino ( 8 fermionic dofs). It is formulated in the superspace, but there are no auxiliary fields since the multiplet is on-shell. An $\mathrm{N}=2$ superparticle in 10 dimensions describes the on-shell modes of $\mathrm{N}=2$ supergravity, namely 64 bosonic and 64 fermionic degrees of freedom.

\footnotetext{
${ }^{2}$ It is important to mention that the superparticle can be quantized using the light-cone gauge. In that case the spectrum can be easily computed and tree level computations can be performed [15. However, there are several limitations to go beyond this point because of lacking of Lorentz covariance.

${ }^{3}$ In the case of $\mathrm{N}=2 \mathrm{~d}=10$ supergravity there are two possibilities: type IIA with $F_{2}, F_{4}$ and type IIB with $F_{1}, F_{3}, F_{5}^{+}$(where the last form is selfdual).
} 
Whereas string theory can be consistently formulated only in 10 dimensions, superparticle models can be formulated also in lower dimensions. ${ }^{4}$ These models in lower dimensions are easier to be used since the BRST conditions for physical states does not put the theory on shell and there is a wider range of consistent backgrounds (vacua).

For what concerns the interactions we have to recall that the superparticle as well as superstrings couple to their own background. This means, for instance, that $\mathrm{N}=2 \mathrm{~d}=4$ superparticle couples to $\mathrm{N}=2 \mathrm{~d}=4$ supergravity. In Sec. 3.3 this is described in detail. More important, we have to underline that $\mathrm{N}=2 \mathrm{~d}=4$ supergravity is characterized by a graviton, two gravitinos and a RR field (known as graviphoton in the literature) and the coupling with $\mathrm{N}=2 \mathrm{~d}=4$ superparticle is dictated by the BRST symmetry In addition, since for the $d=4$ model the supergravity does not need to be on-shell we can choose to set to zero all background fields except the RR field.

We will show later how the RR fields lead to deformations of anticommutative structure of superspace. But before describing this result, it is worth to say few words about superspace.

Let us remind the reader that superspace [29] is a powerful technique to handle supersymetric theories, it is characterized by the bosonic coordinates of manifold and a set of Grassmann coordinates in the spinor rapresentation of the Lorentz group. The superspace technique provides 1) a very compact way to write the equations of motion for the entire supersymmetric multiplet, 2) an extremely economic way to compute Feynamn diagrams taking into account supersymmetry and, 3) a guideline to construct effective actions of supersymmetric theories. Finally, superspace is naturally embedded in the Pure Spinor formulation of string theory.

Now, we are finally in the position to study the supersymmetric analog of noncommutative geometry of bosonic theory (1.1). At this time, we study the deformation of the anticommutator between fermionic coordinates $\theta^{\alpha}$ 35, 36]. We have to recall that there are several studies in that direction [37, 38, 39] where the second-class constrained were used to show that there is a fundamental non-commutative superspace in the quantization of superparticle. This is reviewed in this new formulation, for two reasons: 1) the pure spinor quantization method is the only consistent way to quantize superparticle without losing the super-Poincaré invariace; 2) it is show the role of the RR fields in the present analysis in Sections 4 and 4.1 .

However, only recently [40, 41, 42], using the pure spinor formulation of string theory it is shown how the RR fields deform the anticommutation relations as follows

$$
\left\{\theta^{\alpha}, \theta^{\beta}\right\}=\alpha^{\prime 2} F^{\alpha \beta},
$$

where $F^{\alpha \beta}$ are the $\mathrm{RR}$ fields of $\mathrm{N}=2$ supergravity. It is discussed the implications of these new anticommutator relations on quantum field theory in paper [43] and in the several papers that followed it. The relation (1.3) can also be derived in the context of quantum mechanics, or better in the context of superparticle and this is the purpose of this note. We show that in order to reproduce the relation (1.3) we have to use a peculiar type of superparticle known as $D 0$-brane. It is used again the pure spinor formulation and it is shown that the quantization of the D0 brane leads to non-commutativity in the superspace.

Here, we also discuss the perturbation theory and the limit where the RR fields can be taken either very weak or very strong. Some interesting results emerge from this preliminary analysis and further developments will be discussed elsewhere [21].

Finally, we would like to point out that after the original papers [40, 41, numerous studies followed them and there is now a wide literature on the applications. Since we are not discussing

\footnotetext{
${ }^{4}$ Recently, it has been discovered that also superstrings can be formulated in lower dimensions $30,31,32,33$ and these models can be viewed either as the uncompactified part of 10 dimensional superstrings or as the non-Liouville sector of non-critical superstrings 34 .
} 
the applications to gauge theories or the geometrical implications of the deformed commutation relations in bosonic and fermionic case, we will not include this part of the literature in our references.

\section{A Particle in a Magnetic Field}

We briefly review the formulation of a non-relativistic massive and charged particle in presence of magnetic field. We will do it in a generic dimension and the position of the particle is described by its coordinates $x^{m}$ (with $m=1, \ldots, d$ ). We introduce a constant background $B_{m n}=-B_{n m}$, thus, the action reads $\left(\dot{A}=\partial_{\tau} A\right)$

$$
S=\int d \tau\left(\Pi_{m} \dot{x}^{m}-\frac{1}{2 m} \Pi_{m} \Pi^{m}+B_{m n} \dot{x}^{m} x^{n}\right) .
$$

The indices are raised and lowered with the flat metric $\eta_{m n}$. The conjugate momentum $P_{m}$ can be easily computed and it gives $P_{m}=\Pi_{m}+B_{m n} x^{n}$. Now, if we impose the quantization rules $\left[P_{m}, x^{n}\right]=i \delta_{m}^{n}$, we have that

$$
\left[x^{m}, x^{n}\right]=0, \quad\left[\Pi_{m}, x^{n}\right]=i \delta_{m}^{n}, \quad\left[\Pi_{m}, \Pi_{n}\right]=i B_{m n} .
$$

The other equations of motion are

$$
m \dot{x}^{m}=\Pi^{m}, \quad \dot{\Pi}^{m}+B_{n}^{m} \dot{x}^{n}=0 .
$$

For this equations, it follows that $\dot{P}_{m}=0$. Therefore, in order to take the limit $m \rightarrow 0$, we have to impose the constraints

$$
\Pi^{m} \approx 0,
$$

which are second-class constraints. They have to be treated using the Dirac brackets (see [45] for the definition of Dirac brackets) and this leads to the non-commutation relations for the coordinates

$$
\left[x^{m}, x^{n}\right]_{D}=\left(B^{-1}\right)^{m n},
$$

where the subscript denotes the Dirac brackets.

This model is interesting for three aspects: 1) it represents a simple solvable model of a particle moving in a non-trivial background; 2) it gives the non-commutative relations between the coordinates of the spacetime and, finally 3) it requires Dirac brackets for its quantization. All these ingredients will be found again in the subsequent sections.

\section{$3 \quad$ Superparticles and the BRST symmetry}

\subsection{Action and $\kappa$-symmetry}

We use Dirac basis for gamma matices, and the spacetime is taken to be 4 dimensional. The field content is represented by the bosonic coordinates $x^{m}$ where $m=0, \ldots, 3$, two anticommuting Dirac spinors $\theta_{L}^{\alpha}, \theta_{R}^{\alpha}$ with $\alpha=1, \ldots, 4$ and their conjugate momenta $P_{m}, p_{L \alpha}$ and $p_{R \alpha}$. Since we are considering an $\mathrm{N}=2$ model, we have introduced the notation $L / R$ to distinguish between the two flavours of the spinors. In the case of $d=4$, there is no distinction between type IIA/B since the theory is not chiral in the present case. The Dirac matrices $\gamma_{\alpha \beta}^{m}$ are the usual $4 \times 4$ matrices and satisfy the Fierz identities $\gamma_{m,(\alpha \beta} \gamma_{\gamma) \delta}^{m}=0$. 
Let us consider the superparticle action [11, 12, 13]

$$
S=\int d \tau\left(P_{m} \Pi^{m}-\frac{e}{2} P_{m} P^{m}\right)
$$

in the first order formalism with

$$
\Pi^{m}=\dot{x}+\frac{i}{2} \theta_{L} \gamma^{m} \dot{\theta}_{L}+\frac{i}{2} \theta_{R} \gamma^{m} \dot{\theta}_{R}
$$

This action is invariant under the $\kappa$-symmetry and under the reparametrization of the worldline

$$
\begin{gathered}
\delta \theta_{L}^{\alpha}=\left(P \kappa_{L}\right)^{\alpha}, \quad \delta \theta_{R}^{\alpha}=\left(P \kappa_{R}\right)^{\alpha}, \quad \delta P^{m}=0 \\
\delta x^{m}=\zeta P^{m}+\frac{i}{2}\left(\theta_{L} \gamma^{m} P \kappa_{L}+\theta_{R} \gamma^{m} P \kappa_{R}\right), \\
\delta e=\dot{\zeta}+2 i\left(\dot{\theta}_{L}^{\alpha} \kappa_{L \alpha}+\dot{\theta}_{R}^{\alpha} \kappa_{R \alpha}\right) .
\end{gathered}
$$

where $\kappa_{L / R}$ are the infinitesimal gauge parameters of $\kappa$-symmetry and $\zeta$ is the parameter for diffeomorphisms.

From the action (3.1), we deduce the fermionic constraints

$$
\begin{gathered}
d_{L \alpha}=p_{L \alpha}+\frac{i}{2} P_{m}\left(\gamma^{m} \theta_{L}\right)_{\alpha} \approx 0, \\
d_{R \alpha}=p_{R \alpha}+\frac{i}{2} P_{m}\left(\gamma^{m} \theta_{R}\right)_{\alpha} \approx 0
\end{gathered}
$$

which satisfy

$$
\left\{d_{L \alpha}, d_{L \beta}\right\}=P_{m} \gamma_{\alpha \beta}^{m}, \quad\left\{d_{R \alpha}, d_{R \beta}\right\}=P_{m} \gamma_{\alpha \beta}^{m}, \quad\left\{d_{L \alpha}, d_{R \beta}\right\}=0 .
$$

The last equations are obtained using the canonical commutation relations $\left[P_{m}, x^{n}\right]=i \eta_{m}^{n}$, $\left\{p_{L \alpha}, \theta_{L}^{\alpha}\right\}=-i \delta_{\alpha}^{\beta},\left\{p_{R \alpha}, \theta_{R}^{\beta}\right\}=-i \delta_{\alpha}^{\beta}$.

We have to notice the following facts: 1) there are first- and second-class constraints generated by the operators $d_{L \alpha}$ and $d_{R \alpha}$, and they cannot be disentangled without breaking Lorentz covariance, so the technique of Dirac backets cannot be used here, 2) the second-class constraints of the superparticle have been used in [37, 38, 39] to derive non-(anti)commutation relations among the fermionic coordinates of the superspace. However, since as it stands the superparticle model cannot be quantized we use the Pure-Spinor Formulation.

\subsection{Quantization}

We briefly review the Pure-Spinor formulation of superparticle [17, 18, 19].

We introduce the commuting spinors $l_{L}^{\alpha}$ and $l_{R}^{\alpha}$, which satisfy the pure spinor conditions

$$
l_{L} \gamma^{m} l_{L}=0, \quad l_{R} \gamma^{m} l_{R}=0,
$$

and their conjugate momenta $w_{L \alpha}, w_{R \alpha}$.

We define the BRST operators

$$
Q_{L}=l_{L}^{\alpha} d_{L \alpha}, \quad Q_{R}=l_{R}^{\alpha} d_{R \alpha}
$$


They have the usual form ghost $\times$ constraint. Due to pure spinor constraints (3.6), they are nilpotent up to the gauge transformations of $w_{L \alpha}, w_{R \alpha}$ with the local parameters $\mathrm{E}_{L}$ and $\mathrm{E}_{R}$ given by

$$
\delta w_{L \alpha}=\mathrm{E}_{L m}\left(\gamma^{m} l_{L}\right)_{\alpha}, \quad \delta w_{R \alpha}=\mathrm{E}_{R m}\left(\gamma^{m} l_{R}\right)_{\alpha} .
$$

These gauge transformations remove the degrees of freedom from the spinors $w_{L \alpha}$ and $w_{R \alpha}$ to match those of the pure spinors $l_{L}^{\alpha}$ and $l_{R}^{\alpha}$. Following the usual prescription of the BRST quantization rules, we can define the quantum action as follows [46]

$$
S_{0}=\int d \tau\left(P_{m} \Pi^{m}-\frac{1}{2} P_{m} P^{m}\right)+Q_{L} \int d \tau w_{L \alpha} \dot{\theta}_{L}^{\alpha}-Q_{R} \int d \tau w_{R \alpha} \dot{\theta}_{R}^{\alpha}
$$

Even if it seems the usual BRST procedure, we have to notice that the BRST operators $Q_{L}$ and $Q_{R}$ are nilpotent only up to gauge transformations (3.8). This compensates the fact that the Brink-Schwarz superparticle action (3.1) is not invariant under the BRST transformations. In addition, we can always add to the action BRST invariant terms. The reparametrization is fixed by the gauge condition $e=1$, and we have to add the corresponding ghosts $\int d \tau b \dot{c}$. However, there is no procedure to get (3.9) from an honest gauge fixing of the action (3.1) (a suggestion how this might work is given in [46, 47]).

By exploiting the different contributions in (3.9), we obtain

$$
S_{0}=\int d \tau\left(\dot{\theta}_{L}^{\alpha} p_{L \alpha}+\dot{\theta}_{R}^{\bar{\alpha}} p_{R \bar{\alpha}}+P_{m} \dot{x}^{m}-\frac{1}{2} P_{m} P^{m}-w_{L \alpha} \dot{l}_{L}^{\alpha}-i_{R}^{\alpha} w_{R \alpha}\right)
$$

which is BRST invariant and invariant under the gauge transformation (3.8) if the spinors $l_{L}^{\alpha}, l_{R}^{\alpha}$ are pure. The action is also invariant under supersymmetry transformations generated by $Q_{\epsilon}=\epsilon_{L}^{\alpha} q_{L \alpha}+\epsilon_{R}^{\alpha} q_{R \alpha}$ where

$$
q_{L \alpha}=p_{L \alpha}-\frac{i}{2} P_{m}\left(\gamma^{m} \theta_{L}\right)_{\alpha}, \quad q_{R \alpha}=p_{R \alpha}-\frac{i}{2} P_{m}\left(\gamma^{m} \theta_{R}\right)_{\alpha},
$$

which anticommute with the BRST operators $Q_{L}$ and $Q_{R}$.

The physical states are identified with the BRST cohomology at ghost number 1 and the cohomology is computed by the following equations

$$
Q_{L}\left|\psi>=0, Q_{R}\right| \psi>=0, \quad\left|\psi>\neq Q_{L}\right| \Omega_{L}>+Q_{R} \mid \Omega_{R}>
$$

with $Q_{R}\left|\Omega_{L}>=Q_{L}\right| \Omega_{R}>=0$. The physical state $\mid \psi>$ has ghost number one and the parameters of the gauge transformations $\mid \Omega_{L / R}>$ have ghost number zero. The states $|\psi\rangle$ are obtained by acting with normal-ordered combinations of operators $x^{m}, \theta^{\alpha}, \ldots \ldots$ on the vacuum $\mid 0>$. The complete analysis of eqs. (3.12) in $\mathrm{d}=10 \mathrm{~N}=2$ case has been given in [48, 49, and based on those results it can be shown that the solution of these equations yields the off-shell multiplet of $\mathrm{N}=2 \mathrm{~d}=4$ supergravity.

\subsection{Coupling the superparticle to the background}

As illustrated in [19] the superparticle $N=2$ can be coupled to a $N=2$ supergravity background. The deformation of the action $S+\int d \tau V$ has to be BRST invariant in order to define gauge invariant correlation functions. For constant backgrounds, the BRST invariant action is given by

$$
S_{R}=S_{0}+\int d \tau\left(P^{m} g_{m n} P^{n}+B_{m n} L^{m n}+\Psi_{L m}^{\alpha} q_{L \alpha} P^{m}++q_{R \bar{\alpha}} P^{m} \Psi_{L m}^{\alpha}+i q_{L \alpha} F^{\alpha \beta} q_{R \beta}\right),
$$


where $g_{m n}$ is the usual metric deformation, $B_{m n}$ is the NS-NS two form, $\Psi_{L m}^{\alpha}$ and $\Psi_{R m}^{\alpha}$ are the gravitinos and $F^{\alpha \beta}$ are the R-R field strengths. The fields $P_{m}, q_{L \alpha}, q_{R \bar{\alpha}}$ and

$$
L^{m n}=P^{[m} x^{n]}+\frac{1}{2} p_{L} \gamma^{m n} \theta_{L}+\frac{1}{2} p_{R} \gamma^{m n} \theta_{R}+\frac{1}{2} w_{L} \gamma^{m n} l_{L}+\frac{1}{2} w_{R} \gamma^{m n} \theta_{R},
$$

are BRST invariant. As a consequence, the action (3.13) is invariant if the backgrounds are constant. Given that, we can obtain the action (3.13) by an equation similar to (3.9) (see [46, 19]). In the following we will set all background fields to zero except for the RR graviphoton and the metric $G_{m n}$ and we will take them to be constant.

The advantage of working in 4 dimensions is due to weaker constraints to which the background has to satisfy and the absence of backreaction. Indeed, as is been shown in [31, in $d=4$ the BRST cohomology implies only that the background fields belong to off-shell supermultiplets and no equations of motion are necessary (see also [20]). In the case of closed superstrings 21, it can be shown that the BRST conditions implies only some kinematical restrictions on the background. For that reason, one can choose suitable background enforcing the absence of the backreaction.

\section{$4 \quad$ RR fields and Non-commutative Superspace I}

Setting the background fields $B_{m n}$ and the gravitinos $\Psi_{L m}^{\alpha}, \Psi_{R m}^{\bar{\alpha}}$ to zero and we assume that $F^{\alpha \beta}=F^{\beta \alpha}$, we obtain the new action

$$
\begin{gathered}
S_{R}=\int d \tau\left[\dot{\theta}_{L}^{\alpha} p_{L \alpha}+\dot{\theta}_{R}^{\alpha} p_{R \alpha}+i\left(p_{L \alpha}-\frac{i}{2} P_{m}\left(\gamma^{m} \theta_{L}\right)_{\alpha}\right) F^{\alpha \beta}\left(p_{R \beta}-\frac{i}{2} P_{m}\left(\gamma^{m} \theta_{R}\right)_{\beta}\right)\right] \\
+\int d \tau\left[P_{m} \dot{x}^{m}-\frac{1}{2} G_{m n} P^{m} P^{n}-w_{L \alpha} i_{L}^{\alpha}-i_{R}^{\alpha} w_{R \alpha}\right]
\end{gathered}
$$

where $G_{m n}=\eta_{m n}+g_{m n}$. The presence of RR fields breaks the supersymmetry. The amount of supersymmetry preserved in this background is given by the equations

$$
P_{m} F^{\alpha \beta} \gamma_{\beta \gamma}^{m} \epsilon_{R}^{\gamma}=0, \quad P_{m} F^{\alpha \beta} \gamma_{\alpha \gamma}^{m} \epsilon_{L}^{\gamma}=0
$$

which are the usual Killing equations for spinors if one redefines the supersymmetry parameters with $\epsilon_{L}^{\prime}=P \epsilon_{L}$ and $\epsilon_{R}^{\prime}=P \epsilon_{R}$, for off-shell momentum $P_{m}$. No contribution is added to the ghost action and this simplifies the analysis.

From the action (4.1) we can derive the equations of motion for $\theta_{L}^{\alpha}$ and $\theta_{R}^{\alpha}$

$$
-\dot{\theta}_{L}^{\alpha}+i F^{\alpha \beta} p_{R \bar{\beta}}+\frac{1}{2}(F P)_{\beta}^{\alpha} \theta_{R}^{\beta}=0, \quad-\dot{\theta}_{R}^{\alpha}-i F^{\beta \alpha} p_{L \beta}-\frac{1}{2}(F P)_{\beta}^{\alpha} \theta_{L}^{\beta}=0,
$$

which can be solved in terms of $p_{L \alpha}$ and $p_{R \bar{\alpha}}$. We assume for the time being that $F^{\alpha \beta}$ is an invertible matrix. On the contrary, if $F$ is not invertible on the spinor space, one can decompose any spinor into a part belonging to $\operatorname{ker}(F)$ and to $\operatorname{ker}(F)^{\perp}$. The spinors belonging to the kernel of $\mathrm{F}$ do not enter the coupling term in (4.1) and therefore can be treated separately, in that case there is a residual supersymmetry.

Notice that the RR field $F^{\alpha \beta}$ plays the role of the mass in the case of non-relativistic charge particle in Sec. 2. Therefore, we are interested in studying the limit $\|F\| \rightarrow \infty$, where $\|\cdot\|$ means a measure of the intensity of the RR field strength. ${ }^{5}$ Moreover, the RR fields does not

\footnotetext{
${ }^{5}$ The relation between lowest Landau levels and RR fields is explored in [50]
} 
seem to play the role of the magnetic field $B_{m n}$ of Sec. 2. Indeed, the anticommutation relations among the fermionic constraints $d_{\alpha L}$ and $d_{\alpha R}$ do not contain the field $F^{\alpha \beta}$ in contrast to the corresponding constraints $\Pi^{m}$ of the bosonic case.

To exploit the analogy between the mass term of (2.1) and the RR-dependent terms of (4.1), we further manipulate the action. Substituting (4.3) in the action (4.1), one obtains

$$
S_{R}=\int d \tau\left[i \dot{\theta}_{L}^{\alpha} F_{\alpha \beta}^{-1} \dot{\theta}_{R}^{\beta}+\frac{i}{2} \theta_{L} P \dot{\theta}_{L}+\frac{i}{2} \dot{\theta}_{R} P \theta_{R}\right]+\int d \tau\left[P^{m} \dot{x}^{m}-\frac{1}{2} P^{m} P_{m}-w_{L \alpha} i_{L}^{\alpha}-i_{R}^{\alpha} w_{R \alpha}\right] .
$$

The first term is a kinetic term for the fermions which is quadratic in the derivatives. This is an usual term for spinors, but it is always present in string models in superspace. The second and the third term in the action resemble the spinorial part of the Brink-Schwarz action.

This action suggests that this is a superparticle moving on a supergroup manifold with coordinates $x^{m}, \theta_{L}^{\alpha}$ and $\theta_{R}^{\bar{\alpha}}$. Denoting by $\mathcal{Q}_{L \alpha}, \mathcal{Q}_{R \bar{\alpha}}$ and by $\mathcal{P}_{m}$ the abstract generators of the algebra $\left\{\mathcal{Q}_{L \alpha}, \mathcal{Q}_{L \beta}\right\}=-\gamma_{\alpha \beta}^{m} \mathcal{P}_{m}$ and $\left\{\mathcal{Q}_{R \alpha}, \mathcal{Q}_{R \beta}\right\}=-\gamma_{\alpha \beta}^{m} \mathcal{P}_{m}$, we find the following $\mathrm{MC}$ forms

$$
g^{-1} d g=\left(d x^{m}+d \theta_{L} \gamma^{m} \theta_{L}+d \theta_{R} \gamma^{m} \theta_{R}\right) \mathcal{P}_{m}+d \theta_{L}^{\alpha} \mathcal{Q}_{L \alpha}+d \theta_{R}^{\alpha} \mathcal{Q}_{R \alpha},
$$

and the metric for the algebra have the following non-vanishing entries

$$
\left(\mathcal{P}_{m}, \mathcal{P}_{n}\right)=g_{m n}, \quad\left(\mathcal{Q}_{R \alpha}, \mathcal{Q}_{L \beta}\right)=F_{\alpha \beta}^{-1} .
$$

Notice that the MC forms are not supersymmetric invariant since the supersymmetry is broken by the presence of the RR fields. Eliminating $P_{m}$ from the action (4.4) and using the metric given in (4.6), the action (4.4) can be written as

$$
S_{R}=\int d \tau\left[\left(g^{-1} \dot{g}, g^{-1} \dot{g}\right)-w_{L \alpha} l_{L}^{\alpha}-l_{R}^{\bar{\alpha}} w_{R \bar{\alpha}}\right]
$$

This is similar to the result found in [51, where the authors showed that in the case of $A d S_{3} \times S^{3}$, one finds a sigma model on a supergroup. It is interesting that the same situation is reproduced in the present context (it should also be possible to do it for string theory in 10 dimension with constant RR fluxes as studied in [52, 53, 40, but nobody found a convenient set of variables yet).

After the conjugated momenta $p_{\alpha L / R}$ are removed, the equations of motion for the spinors read

$$
\ddot{\theta}_{L}^{\alpha}+\frac{1}{2} F^{\alpha \bar{\beta}} P_{\bar{\beta} \bar{\gamma}} \dot{\theta}_{R}^{\bar{\gamma}}=0, \quad \ddot{\theta}_{R}^{\bar{\alpha}}-\frac{1}{2} \dot{\theta}_{L}^{\gamma} P_{\gamma \beta} F^{\beta \bar{\alpha}}=0 .
$$

Since on-shell we have $\dot{P}_{m}=0$ and since we choose constant RR field strengths, we can integrate once the above equations to get

$$
\dot{\theta}_{L}^{\alpha}+\frac{1}{2} F^{\alpha \bar{\beta}} P_{\bar{\beta} \bar{\gamma}} \theta_{R}^{\bar{\gamma}}=C_{L}^{\alpha}, \quad \dot{\theta}_{R}^{\bar{\alpha}}-\frac{1}{2} F^{\bar{\alpha} \beta} P_{\beta \gamma} \theta_{L}^{\gamma}=C_{R}^{\bar{\alpha}}
$$

where $C_{L / R}$ are integration constants to be fixed by boundary conditions (in order to avoid any new constant non-covariant quantity we choose to set them to zero). Inserting (4.9) into (4.8), we arrive at the decoupled equations for $\theta_{L}^{\alpha}$ and $\theta_{R}^{\bar{\alpha}}$

$$
\ddot{\theta}_{L}^{\alpha}-\frac{1}{4}\left(F P F^{T} P\right)_{\beta}^{\alpha} \theta_{L}^{\beta}=0, \quad \ddot{\theta}_{R}^{\bar{\alpha}}-\frac{1}{4} \theta_{R}^{\bar{\beta}}\left(P F^{T} P F\right)_{\bar{\beta}}^{\bar{\alpha}}=0 .
$$

These equations show that the RR fields play the role of a mass term for the fundamental fields $\theta^{\alpha}$ and the matrix $\left(F \not P F^{T} \not P\right)$ is constant. This is a well-known phenomena in $p p$-waves 
background [54, 55]: the spectrum of worldsheet theory becomes massive. In addition, we can see that the theory is not supersymmetric: the bosonic partner is not massive. ${ }^{6}$

Nevertheless, if the matrix $\left(F \not P F^{T} \not P\right)$ has some zero eigenvalues, along those directions we recover a partial supersymmetry.

Let us study two interesting limits: $\|F\| \rightarrow 0$ and $\|F\| \rightarrow \infty$. The first limit is the regime where perturbation theory can be used to perform worldline computations. The second limit is certainly more interesting due to the fact that very little is know about string theory in the presence of strong background fields.

In the limit $\|F\| \rightarrow \infty$, where the norm $\|\cdot\|$ is properly defined, the first term can be neglected, and the action is invariant under a new $\kappa$-symmetry (notice that we have replaced the classical $\kappa$-symmetry of the action (3.1) with the BRST symmetry given in eq. (3.7))

$$
\begin{gathered}
\delta_{k} x^{m}=\theta_{L} \gamma^{m} P \kappa_{L}+\theta_{R} \gamma^{m} P \kappa_{R}+\zeta P^{m}, \\
\delta_{k} \theta_{L}^{\alpha}=-(P \kappa)_{L}^{\alpha}+\zeta \dot{\theta}_{L}^{\alpha}, \quad \delta_{k} \theta_{R}^{\bar{\alpha}}=-(P \kappa)_{R}^{\bar{\alpha}}+\zeta \dot{\theta}_{R}^{\bar{\alpha}},
\end{gathered}
$$

where the diffeomorphism ghost $\zeta$ compensates the gauge choice $e=1$ by choosing the following solution $\zeta=-\int^{\tau} d \tau^{\prime}\left(\dot{\theta}_{L}^{\alpha} \kappa_{L \alpha}+\dot{\theta}_{R}^{\bar{\alpha}} \kappa_{R \bar{\alpha}}\right)$.

Notice also that from eqs. (4.3) it turns out that to take properly the limit of $\|F\| \rightarrow \infty$ one has to impose the constraints

$$
q_{\alpha L} \approx 0, \quad q_{\alpha R} \approx 0
$$

These constraints generate the $\kappa$-symmetry (4.11) with the opposite sign is front of the transformation rules for the spinors. In addition, they include also second-class constraints. The theory is quantized and therefore we can use the technique discussed in [12, 37, 38. This yields the same non-(anti) commutative superspace which is a consequence of the structure of second-class constraints of $q_{\alpha L / R}$ and it does not depend upon the RR field. Applying the Dirac procedure, one finds that 37,38

$$
\left\{\theta_{R}^{\alpha}, \theta_{R}^{\beta}\right\}_{D} \sim \gamma_{m}^{\alpha \beta} x^{m}, \quad\left\{\theta_{L}^{\alpha}, \theta_{L}^{\beta}\right\}_{D} \sim \gamma_{m}^{\alpha \beta} x^{m}, \quad\left\{\theta_{L}^{\alpha}, \theta_{R}^{\beta}\right\}_{D}=0
$$

This result for the superparticle is quite different from the result of superstrings [40, 41, 43] given in (1.3); for the superparticle, the anticommutation relations of the Grassmann coordinates (4.13) are related to the bosonic coordinates and not the RR field. This is due to the fact that we have only one set of free parameters, namely $F^{\alpha \beta}$, which have to be interpreted as a mass matrix and not as a "magnetic field".

Moreover, for highly curved space one has to take into account the radiative corrections to the action (4.4) before taking the limit $\|F\| \rightarrow \infty$. In fact, as we shall show it below, at one loop there are new pieces generated by radiative correction at one-loop in the worldline.

On the other hand, in the limit $\|F\| \rightarrow 0$, the first term becomes dominant over the other fermionic terms (for the bosonic terms in (4.4), one can also add a background metric $-\frac{1}{2} g^{m n} P_{m} P_{n}$ and therefore they cannot be neglected) and the action (4.4) with background metric $g_{m n}$ reduces to

$$
S_{R}=\int d \tau\left[-\dot{\theta}_{R}^{\bar{\alpha}} F_{\bar{\alpha} \beta}^{-1} \dot{\theta}_{L}^{\beta}+\frac{1}{2} g_{m n} \dot{x}^{n} \dot{x}^{m}+w_{L \alpha} \dot{l}_{L}^{\alpha}+i_{R}^{\bar{\alpha}} w_{R \bar{\alpha}}\right] .
$$

\footnotetext{
${ }^{6}$ It has to be recalled that the mass parameter in a curved space does not carefully measure the masslessness of the field. The best way to reveal a supersymmetry breaking is to analyze the Killing spinor equations in a curved background.
} 
We conclude that we can use the RR background to set up a perturbation theory around weak RR backgrounds. Namely, we can consider the action (4.14) as the quadratic part of the action from which the propagators can be computed, and the rest has to be considered as a perturbation.

\subsection{Radiative Corrections}

It is easy to compute the radiative corrections to the bosonic inverse propagator $\left\langle x^{m}(\tau) x^{n}(0)\right\rangle$. By computing the free propagators of $x^{m}$ and the off-diagonal propagator of $\theta_{L}$ and $\theta_{R}$, one can obtain the one-loop contribution

$$
G^{m n}(\tau)=-\frac{1}{16} \gamma_{\alpha \beta}^{m} F^{\beta \bar{\gamma}} \gamma_{\bar{\gamma} \bar{\delta}}^{n} F^{\alpha \bar{\delta}} \int_{0}^{1} d t|\tau-t||t|=-\frac{1}{16} \operatorname{Tr}\left(\gamma^{m} F \gamma^{n} F\right) \mathcal{P}(\tau)
$$

where $\mathcal{P}(\tau)$ is a polynomial of third order in $\tau$. In the same way the one-loop corrections to the off-diagonal inverse propagator $\left\langle\theta_{L}^{\alpha} \theta_{R}^{\bar{\beta}}\right\rangle$

$$
G_{\alpha \bar{\beta}}(\tau)=-\frac{1}{16} \gamma_{\alpha \delta}^{m} F^{\delta \bar{\gamma}} \gamma_{\bar{\gamma} \bar{\beta}}^{n} g_{m n} \mathcal{P}(\tau)
$$

Form this computation, we see how the presence of RR backgrounds in the $\left\langle\theta_{L}^{\alpha} \theta_{R}^{\bar{\beta}}\right\rangle$ leads to modifications in all the couplings and therefore the analysis at string coupling $\|F\| \rightarrow \infty$ cannot be performed without taking into account the radiative corrections. It may be possible to re-sum all the contributions. Notice for example that the spinors $\theta_{L}$ and $\theta_{R}$ appear only quadratically in (4.14), therefore one can integrate over those fields obtaining the following determinant

$$
\operatorname{det}\left(\begin{array}{cc}
\frac{i}{2} P_{\alpha \beta} \partial_{\tau} & \frac{1}{2} F_{\alpha \bar{\beta}}^{-1} \partial_{\tau}^{2} \\
\frac{1}{2} F_{\bar{\alpha} \beta}^{-1} \partial_{\tau}^{2} & \frac{i}{2} P_{\bar{\alpha} \bar{\beta}} \partial_{\tau}
\end{array}\right) .
$$

The next step will be to integrate over the field $P_{m}$. We will not pursue this analysis here and we refer to a subsequent publication [21].

\section{Do-branes and the BRST symmetry}

Since we have seen that the introduction of the RR fields in the case of $N=2 d=4$ (or $d=10$ ) superparticle does not really help for deriving the anticommutation relation (1.3) for the Grassmann coordinates, we need to use a different type of superparticle to do it.

As is known, in string theory there are solitonic degrees of freedom which are known as Dp-branes [26]. A given Dp-brane has a worldvolume which has $p+1$ dimensions. They are characterized by the coupling to the RR fields of the superstrings, and they can be described by a low energy effective action which is the sum of a Born-Infeld action and a Wess-Zumino term. Among the Dp-branes, we can consider the D0-brane which is a particle (the worldvolume is 1-dimensional) described by the effective action

$$
S=\int d \tau\left(P_{m} \Pi^{m}-\frac{e}{2}\left(P_{m} P^{m}+f^{2}\right)\right)+\int d \tau f_{\alpha \beta} \theta_{L}^{\alpha} \dot{\theta}_{R}^{\beta}
$$

in the first order formalism with

$$
\Pi^{m}=\dot{x}+\frac{i}{2} \theta_{L} \gamma^{m} \dot{\theta}_{L}+\frac{i}{2} \theta_{R} \gamma^{m} \dot{\theta}_{R}
$$


and $f_{\alpha \beta}$ is constant. (In the case of $\mathrm{d}=10$ action, $f_{\alpha \beta}$ is a scalar proportional to $\delta_{\alpha}^{\beta}$.) The first term is the Born-Infeld term written in the first order formalism. The second term is a Wess-Zumino term and the coefficient $f_{\alpha \beta}$ is related to the brane tension [56]. Indeed it can be viewed as the mass of the $D 0$-brane

The action is invariant under $\kappa$-symmetry which yields the supersymmetry of the $D 0$-brane and it can be quantized using the BRST technique discussed above in Sec. 3.2. An objection to this might be: the $D 0$-brane is not a fundamental degree of freedom and there is no need of quantizing the it! However it is shown in 44] that the BRST based on Pure Spinor formulation replaces the $\kappa$-symmetry and provides a guideline how to coupled the $D 0$-brane to a supergravity background as in the case of the $\mathrm{N}=2$ superparticle.

In analogy with eqs. (3.11), we derive the supersymmetry generators $q_{\alpha, L}$ and $q_{\alpha, R}$ for the D0-brane.

$$
\begin{aligned}
& q_{L \alpha}=p_{L \alpha}-\frac{i}{2} P_{m}\left(\gamma^{m} \theta_{L}\right)_{\alpha}+f_{\alpha \beta} \theta_{R}^{\beta}, \\
& q_{R \alpha}=p_{R \alpha}-\frac{i}{2} P_{m}\left(\gamma^{m} \theta_{R}\right)_{\alpha}+f_{\alpha \beta} \theta_{L}^{\alpha} .
\end{aligned}
$$

They couple to the RR field of the supergravity background and the supersymmetry is broken because of the presence of RR fields, or equivalently to the presence of $D 0$-branes.

\section{RR fields and Non-commutative Superspace II}

Finally, we can couple the D0-brane to RR field of the supergravity background and this introduces a new term of the form

$$
\int d \tau F^{\alpha \beta} q_{\alpha L} q_{\beta R}
$$

such as in the case of a superparticle coupled to RR fields. Repeating the derivation as in sec. 4. we find that in the limit of $\|F\| \rightarrow \infty$, we need the constraints

$$
q_{\alpha L} \approx 0, \quad q_{\alpha R} \approx 0 .
$$

where $q_{\alpha L}$ and $q_{\alpha R}$ are given by (5.3) and they depend on the RR field $f_{\alpha \beta}$ generated by the D0-brane. Applying the Dirac procedure and using the canonical brackets $\left[p_{\alpha L / R}, \theta_{L / R}^{\beta}\right]=i \delta_{\alpha}{ }^{\beta}$, we end up with the commutation relations

$$
\left\{\theta_{L}^{\alpha}, \theta_{R}^{\beta}\right\}_{D}=\left(f^{-1}\right)^{\alpha \beta},
$$

which finally gives the non-(anti)commutation relations between the fermionic coordinates.

To conclude, we have shown that the coupling of the RR fields and the Wess-Zumino term, in the case of superparticle and D0-brane, are fundamental to generate a deformation of anti-

commutation relations among Grassmann coordinates of the superspace. It is point out that the RR fields of the supergravity background cannot generate the wanted commutation relations, but it replaces the role of the mass as in the case of the non-relativistic particle moving in a magnetic field. Furthermore, it is shown that the brane tension together with a supergravity background yields the wanted commutation relations (1.3) obtained also in string theory. The role of the mass and and of the RR fields is inverted: in the case of massive bosonic charge particle, we take the limit $m \rightarrow 0$ and we derive (1.1) deformed by $B_{m n}$, in the case of superparticles, we take the limit $\|F\| \rightarrow \infty$ and we derive (1.3) deformed by the brane tension $f$. We argued that this limit can be taken only by neglecting the radiative corrections and a deeper analysis will be presented elsewhere [21]. 


\section{Acknowledgements}

The author would like to thank the organizers and the directors of the $43^{\text {rd }}$ International School on Subnuclear Physics held in Erice, Sicily in Sept. 2005 where a lecture based on the present paper has been delivered. We also thank I. Bars, L. Castellani, A. Lerda, F. Morales, Y. Oz, L. Tamassia and N. Wyllard for useful discussions on related subjects and comments. We thank P. Aschieri for a useful discussion based on work [57] where a wide class of superspace non-commutative deformations are discussed and that stimulated the present derivation of the anti-commutation relations. A special thank to L. Anguelova for a careful reading of the manuscript and for useful suggestions.

\section{References}

[1] C. S. Chu and P. M. Ho, "Noncommutative open string and D-brane," Nucl. Phys. B 550, 151 (1999) arXiv:hep-th/9812219.

[2] C. S. Chu and P. M. Ho, "Constrained quantization of open string in background B field and noncommutative D-brane," Nucl. Phys. B 568, 447 (2000) arXiv:hep-th/9906192.

[3] F. Ardalan, H. Arfaei and M. M. Sheikh-Jabbari, "Noncommutative geometry from strings and branes," JHEP 9902, 016 (1999) arXiv:hep-th/9810072.

[4] N. Seiberg and E. Witten, "String theory and noncommutative geometry," JHEP 9909, 032 (1999) arXiv:hep-th/9908142.

[5] D. Bigatti and L. Susskind, "Magnetic fields, branes and noncommutative geometry," Phys. Rev. D 62, 066004 (2000) arXiv:hep-th/9908056.

[6] R. Peierls, "Zur Theorie des Diamagnetismus von Leitungselektronen," Z. Phys. 80, 763 (1933).

[7] G. V. Dunne, R. Jackiw and C. A. Trugenberger, "Topological' (Chern-Simons) Quantum Mechanics," Phys. Rev. D 41, 661 (1990).

[8] R. Jackiw, "Physical instances of noncommuting coordinates," Nucl. Phys. Proc. Suppl. 108, 30 (2002) [Phys. Part. Nucl. 33, S6 (2002 LNPHA,616,294-304.2003)] arXiv:hep-th/0110057.

[9] H. S. Snyder, "Quantized Space-Time," Phys. Rev. 71, 38 (1947).

[10] C. N. Yang, "On Quantized Space-Time," Phys. Rev. 72, 874 (1947).

[11] R. Casalbuoni, "The Classical Mechanics For Bose-Fermi Systems," Nuovo Cim. A 33, 389 (1976).

[12] L. Brink and J. H. Schwarz, "Clifford Algebra Superspace," CALT-68-813

[13] W. Siegel, "Hidden Local Supersymmetry In The Supersymmetric Particle Action," Phys. Lett. B 128, 397 (1983).

[14] P. A. Grassi, G. Policastro and M. Porrati, "Covariant quantization of the Brink-Schwarz superparticle," Nucl. Phys. B 606, 380 (2001) arXiv:hep-th/0009239.

[15] M. B. Green, J. H. Schwarz and E. Witten, "Superstring Theory. Vol. 1: Introduction," and "Superstring Theory. Vol. 2: Loop Amplitudes, Anomalies And Phenomenology,"

[16] N. Berkovits, "Super-Poincaré covariant quantization of the superstring," JHEP 04 (2000) 018, hep-th/0001035

[17] N. Berkovits, "Covariant quantization of the superparticle using pure spinors," JHEP 0109, 016 (2001) arXiv:hep-th/0105050.

[18] N. Berkovits, "ICTP lectures on covariant quantization of the superstring," hep-th/0209059

[19] N. Berkovits, "Covariant quantization of the supermembrane," JHEP 0209, 051 (2002) arXiv:hep-th/0201151. 
[20] P. A. Grassi and J. F. Morales, "Partition functions of pure spinors," arXiv:hep-th/0510215.

[21] P. A. Grassi and L. Tamassia, in preparation.

[22] P. A. Grassi, G. Policastro, M. Porrati, and P. van Nieuwenhuizen, "Covariant quantization of superstrings without pure spinor constraints," JHEP 10 (2002) 054, hep-th/0112162 P. A. Grassi, G. Policastro, and P. van Nieuwenhuizen, "The massless spectrum of covariant superstrings," JHEP 11 (2002) 001, hep-th/0202123 P. A. Grassi, G. Policastro, and P. van Nieuwenhuizen, "On the BRST cohomology of superstrings with/without pure spinors," Adv. Theor. Math. Phys. 7 (2003) 499, hep-th/0206216

[23] M. Chesterman, "Ghost constraints and the covariant quantization of the superparticle in ten dimensions," JHEP 02 (2004) 011, hep-th/0212261

[24] Y. Aisaka and Y. Kazama, "A new first class algebra, homological perturbation and extension of pure spinor formalism for superstring," JHEP 02 (2003) 017, hep-th/0212316

[25] P. A. Grassi, G. Policastro and P. van Nieuwenhuizen, "The covariant quantum superstring and superparticle from their classical actions," Phys. Lett. B 553, 96 (2003) arXiv:hep-th/0209026.

[26] J. Polchinski, "String theory. Vol. 1: An introduction to the bosonic string," J. Polchinski, "String theory. Vol. 2: Superstring theory and beyond,"

[27] M. B. Green, M. Gutperle and P. Vanhove, "One loop in eleven dimensions," Phys. Lett. B 409, 177 (1997) arXiv:hep-th/9706175. M. B. Green, H. h. Kwon and P. Vanhove, "Two loops in eleven dimensions," Phys. Rev. D 61, 104010 (2000) arXiv:hep-th/9910055.

[28] L. Anguelova, P. A. Grassi and P. Vanhove, Nucl. Phys. B 702, 269 (2004) arXiv:hep-th/0408171.

[29] S. J. Gates, M. T. Grisaru, M. Rocek and W. Siegel, "Superspace, Or One Thousand And One Lessons In Supersymmetry," Front. Phys. 58, 1 (1983) arXiv:hep-th/0108200.

[30] N. Berkovits, "Pure spinor formalism as an $\mathcal{N}=2$ topological string," hep-th/0509120

[31] P. A. Grassi and N. Wyllard, "Lower-dimensional pure-spinor superstrings," arXiv:hep-th/0509140

[32] N. Wyllard, "Pure-spinor superstrings in $\mathrm{d}=2,4,6$," arXiv:hep-th/0509165

[33] O. Chandia, "D = 4 pure spinor superstring and $\mathrm{N}=2$ strings," arXiv:hep-th/0509185

[34] P. A. Grassi and Y. Oz, "Non-critical covariant superstrings," arXiv:hep-th/0507168.

[35] S. Ferrara and M. A. Lledo, "Some aspects of deformations of supersymmetric field theories," JHEP 0005, 008 (2000) arXiv:hep-th/0002084.

[36] D. Klemm, S. Penati and L. Tamassia, "Non(anti)commutative superspace," Class. Quant. Grav. 20, 2905 (2003) arXiv:hep-th/0104190.

[37] L. Brink and J. H. Schwarz, "Quantum Superspace," Phys. Lett. B 100, 310 (1981).

[38] J. H. Schwarz and P. Van Nieuwenhuizen, "Speculations Concerning A Fermionic Substructure Of Space-Time," Lett. Nuovo Cim. 34 (1982) 21.

[39] I. Bars, C. Deliduman, A. Pasqua and B. Zumino, "Superstar in noncommutative superspace via covariant quantization of the superparticle," Phys. Rev. D 68, 106006 (2003) arXiv:hep-th/0308107.

[40] J. de Boer, P. A. Grassi and P. van Nieuwenhuizen, "Non-commutative superspace from string theory," Phys. Lett. B 574, 98 (2003) arXiv:hep-th/0302078.

[41] H. Ooguri and C. Vafa, "The C-deformation of gluino and non-planar diagrams," Adv. Theor. Math. Phys. 7, 53 (2003) arXiv:hep-th/0302109.

[42] N. Berkovits and N. Seiberg, "Superstrings in graviphoton background and N = 1/2 + 3/2 supersymmetry," JHEP 0307, 010 (2003) arXiv:hep-th/0306226.

[43] N. Seiberg, "Noncommutative superspace, $\mathrm{N}=1 / 2$ supersymmetry, field theory and string theory," JHEP 0306, 010 (2003) arXiv:hep-th/0305248. 
[44] L. Anguelova and P. A. Grassi, "Super D-branes from BRST symmetry," JHEP 0311, 010 (2003) arXiv:hep-th/0307260.

[45] M. Henneaux and C. Teitelboim, "Quantization of gauge systems,", Princeton University Press, Princeton, New Jersey.

[46] I. Oda and M. Tonin, "On the Berkovits covariant quantization of GS superstring," Phys. Lett. B 520 (2001) 398 arXiv:hep-th/0109051. M. Matone, L. Mazzucato, I. Oda, D. Sorokin and M. Tonin, "The superembedding origin of the Berkovits pure spinor covariant quantization of superstrings," Nucl. Phys. B 639, 182 (2002) arXiv:hep-th/0206104.

[47] Y. Aisaka and Y. Kazama, "Origin of pure spinor superstring," JHEP 0505, 046 (2005) arXiv:hep-th/0502208.

[48] N. Berkovits and P. S. Howe, "Ten-dimensional supergravity constraints from the pure spinor formalism for the superstring," Nucl. Phys. B 635, 75 (2002) arXiv:hep-th/0112160.

[49] P. A. Grassi and L. Tamassia, "Vertex operators for closed superstrings," JHEP 0407, 071 (2004) arXiv:hep-th/0405072.

[50] M. Hatsuda, S. Iso and H. Umetsu, "Noncommutative superspace, supermatrix and lowest Landau level," Nucl. Phys. B 671, 217 (2003) arXiv:hep-th/0306251.

[51] N. Berkovits, C. Vafa and E. Witten, "Conformal field theory of AdS background with RamondRamond flux," JHEP 9903, 018 (1999) arXiv:hep-th/9902098.

[52] N. Berkovits and O. Chandia, "Superstring vertex operators in an AdS(5) x S(5) background," Nucl. Phys. B 596, 185 (2001) arXiv:hep-th/0009168.

[53] N. Berkovits, "Conformal field theory for the superstring in a Ramond-Ramond plane wave background," JHEP 0204, 037 (2002) arXiv:hep-th/0203248.

[54] R. R. Metsaev, "Type IIB Green-Schwarz superstring in plane wave Ramond-Ramond background," Nucl. Phys. B 625, 70 (2002) arXiv:hep-th/0112044.

[55] R. R. Metsaev and A. A. Tseytlin, "Exactly solvable model of superstring in plane wave Ramond-Ramond background," Phys. Rev. D 65, 126004 (2002) arXiv:hep-th/0202109.

[56] M. Aganagic, C. Popescu and J. H. Schwarz, "Gauge-invariant and gauge-fixed D-brane actions," Nucl. Phys. B 495, 99 (1997) arXiv:hep-th/9612080.

[57] P. Aschieri, M. Dimitrijevic, F. Meyer and J. Wess, "Noncommutative geometry and gravity," arXiv:hep-th/0510059. 